\title{
An Initial, Prospective Exploration of Specific Stressors for Parents of Children Admitted to an Inpatient Psychiatric Unit
}

\section{Elena Hissett ${ }^{1 *}$, Sumru Bilge-Johnson ${ }^{2}$ and Neil $\mathrm{McNinch}^{3}$}

${ }^{1}$ Child and Adolescent Psychiatry Fellow, Akron Children's Hospital, Akron, OH 44311, USA

${ }^{2}$ Program Director, Child and Adolescent Psychiatry Fellowship, Assistant Professor of Child Psychiatry, Northeast Ohio Medical University, Akron Children's Hospital, Akron, $\mathrm{OH} 44311, \mathrm{USA}$

${ }^{3}$ Biostatistician, The Rebecca D.Considine Research Institute, One Perkins Square, Akron, OH 44308,USA

\begin{abstract}
Increased parental stress has a negative effect on a child's symptoms, which worsens pathology. No studies have been conducted looking at parental stress for children admitted to an inpatient psychiatric unit, and an examination of these stressors may lead to interventions which could benefit the recovery of children with mental illness.

Methods: 66 caregivers of children admitted to the psychiatric unit at Akron Children's Hospital took a 10-question survey designed by investigators. Demographic information and chart review was conducted, and an additional standardized instrument evaluating parental stress was given to parents with children under age 12.

Results: The average T-score on the Parental Stress Index Short Form for these parents is 59.5, which is in the 80th percentile for parent-child dysfunctional relationship, which indicates high stress. Parents are stressed most about the child's future, safety of the child and family, balancing the needs of other family members in addition to the child's need for treatment, confusion about the diagnosis, financial aspects, personal emotional health, and stigma.

Conclusions: This pilot study has a sample size of 66 parents and caregivers, which exceeds previous studies. Our results parallel findings of other studies looking at stress in parents of children admitted to medical units as well as children in outpatient mental health treatment. Parents felt significant distress and lack of confidence, and parental education level did not affect concern about stigma or confusion related to their child's diagnosis. These results support an intervention for greater parent psycho-education and peer support prior to their child's discharge.
\end{abstract}

Keywords: Psychiatric; Child behavior; Bipolar disorder; Education

\section{Background}

There have been studies investigating the multiple stressors facing parents of children with psychiatric and behavioral disturbance [1]. Also, it has been demonstrated that increased stress experienced by parents can have a negative effect on a child's symptoms, which in turn leads to ongoing, worsening pathology [2]. Some stressors previously expressed by parents of children with Bipolar Disorder have included "obtaining an appropriate diagnosis, developing a functional treatment plan, and dealing with the myriad of difficult symptoms at home and at school," as well as "stigma and blame" as many of these parents also experienced mood disorders [3]. Parents were also noted to have diminished relationships with friends and relatives, as well as financial, emotional, and physical strain [4]. Focused attention on the child with mental illness also leads to unhealthy self-neglect and neglect of others in the family [5]. Additional stressors included interactions with healthcare professionals where parents felt that professionals did not believe their reports about behavior, or it was suggested that symptoms were due to poor parenting [3]. Studies have been done looking at stressors affecting parents of children admitted to medical units. These studies have led to changes in medical staff interactions with parents and have shown improvements in parental stress and patient care [6]. No studies have been conducted looking at stress affecting parents of children admitted to an inpatient psychiatric unit. One study with parents of bipolar youth in an outpatient setting examined how parental psychiatric education was helpful in reducing anxiety and sense of being overwhelmed by the child's condition [1].

\section{Objectives}

There is increased interest in providing support to these parents as a way to possibly improve children's prognosis. This initial study would therefore be an important exploration of additional opportunities to offer meaningful support and guidance, as well as additional psychoeducation for caregivers of children with mental illness. This study would foreseeably lead to improved prognosis and stability for children with mental illness. Therefore, the primary aim for this study is to investigate the unique stressors effecting parents of children admitted to a child psychiatry unit, and how a child's psychiatric illness can affect parent's stress. This information can later be used for programing aimed at better support and psycho-education of parents of these patients, as well as staff members working on our unit. As many of the patients in our study sample were admitted after a suicide attempt and/or had a history of suicide attempt, we hypothesize that there will be associations between the number of suicide attempts and parents reported concern about the child's future, as well as increased concern based on the child's Axis I diagnosis, and if there has been a family history of mental illness. We predict more stress associated with interactions with mental health professional based on the Axis I diagnosis, and that the parental education level may be conversely associated with greater confusion about the child's diagnosis and concern about stigma.

\section{Methods}

After granting informed consent, 66 primary caregivers of children admitted to the inpatient psychiatric unit at Akron Children's Hospital between February 2013 and February 2014 participated in our data

*Corresponding author: Elena Hissett, Child and Adolescent Psychiatry Fellow Akron Children's Hospital, Akron, OH 44311,USA, Tel: 330-543-8589; Fax: 330-5433856; E-mail: ehissett@chmca.org

Received December 10, 2014; Accepted March 06, 2015; Published March 13 2015

Citation: Hissett E, Bilge-Johnson S, McNinch N (2015) An Initial, Prospective Exploration of Specific Stressors for Parents of Children Admitted to an Inpatient Psychiatric Unit. J Psychol Abnorm Child 4: 138. doi:10.4172/2329-9525.1000138

Copyright: $\odot 2015$ Hissett $E$, et al. This is an open-access article distributed under the terms of the Creative Commons Attribution License, which permits unrestricted use, distribution, and reproduction in any medium, provided the original author and source are credited. 
Citation: Hissett E, Bilge-Johnson S, McNinch N (2015) An Initial, Prospective Exploration of Specific Stressors for Parents of Children Admitted to an Inpatient Psychiatric Unit. J Psychol Abnorm Child 4: 138. doi:10.4172/2329-9525.1000138

Page 2 of 7

collection. This data included a 10 -question survey designed by the authors of this study for parents to rate their stress concerning various factors associated with caring for a child with mental illness. This survey is not a standardized instrument and was constructed by consulting with nurses, social workers as well as a special group of social workers called parent partners who themselves have children with mental illness and work to provide support and resources for parents. We also designed questions based on the findings of Dr. Fristad, $\mathrm{PhD}$ and her colleges $[1,3,5]$, as well as the book Working with Families of the Mentally Ill by Bernheim and Lehman $[7,8]$, as there is no current standardized instrument for parental stress in older children/teens. The survey was validated to determine readability, and has a Flesch-Kincaid Grade Level of 10.2, and an average readability grade level of 9.5. A chart review was also conducted on these 66 patients to collect demographic information, background data concerning their severity of illness, including number of admissions, diagnosis, number of suicide attempts, history of self-harm, number of psychotropics prescribed, presence of legal problems, type of substance use, family psychiatric history and educational problems such as suspensions or expulsions.13 of these parents had children under the age of 12-years-old admitted, so they also participated in an instrument called the Parental Stress Index Short Form, 4th edition, which evaluates parent and child dynamics which contribute to stress and dysfunctional parenting (7). This dysfunctional dynamic between parent and child can then lead to worsening mental health pathology in the child. The PSI-4-SF is validated for multicultural use and internal consistency, with test-retest reliability of 0.84 over 6 months, and validity when correlated with the full length PSI-4 is 0.98 .Statistical analysis was completed by the biostatistician. Chi Square and Fisher's Exact Tests were performed on the categorical variables to examine for potential associations. All testing was done at the alpha $=0.05$ level of significance, using software R 3.0.2.

\section{Results}

Theaverage age of the children is 14.3 years, and the average grade level is 9 th grade. $70 \%$ of parents have at least a college education. There is an average of 2 inpatient admissions with a mean of 1 suicide attempt. The average number of people per household is 5 people.

Tables 1 and 2 detail patient demographics and mental health data. Table 3 shows results for our 10 question survey.

\begin{tabular}{|c|c|c|c|c|c|}
\hline Gender & Male & Female & & & \\
\hline$\%$ & 30.3 & 69.7 & & & \\
\hline Axis I Diagnosis & Mood disorder NOS & Mood disorder & Anxiety disorder & Multiple diagnoses & \\
\hline$\%$ & 19.7 & 39.4 & 10.6 & 30.3 & \\
\hline Substance Use & None & Alcohol & THC & Cigarettes & Combinations \\
\hline$\%$ & 53.0 & 4.5 & 4.5 & 3.0 & 34.8 \\
\hline Socioeconomic status & Lower & Lower-middle & Middle & Upper-middle & \\
\hline$\%$ & 9.5 & 19.0 & 65.1 & 6.3 & \\
\hline People involved in child's care & Adopted, foster care & Single parent & Two parents & Immediate and extended family & \\
\hline$\%$ & 7.6 & 19.7 & 50.0 & 22.7 & \\
\hline Relationship status of bio-parents & Parental Death & Divorced & Separated & Married & \\
\hline$\%$ & 4.7 & 54.7 & 15.6 & 25.0 & \\
\hline
\end{tabular}

Table 1: Patient Information.

\begin{tabular}{|l|c|c|}
\hline & Yes (\%) & No (\%) \\
\hline Axis II Diagnosis & 25.8 \\
\hline Eating D. & 7.6 \\
\hline ADHD & 21.2 & 74.2 \\
\hline Self-Harm & $\mathbf{7 4 . 2}$ & $\mathbf{9 2 . 7}$ \\
\hline Medical Prob. & $\mathbf{2 4 . 2}$ & $\mathbf{7 8 . 8}$ \\
\hline Legal Prob. & $\mathbf{2 5 . 8}$ \\
\hline Individual Education Plan & 13.6 & $\mathbf{7 5 . 8}$ \\
\hline Expulsions and Suspensions & $\mathbf{8 6 . 4}$ \\
\hline Family History of Mental D. & $\mathbf{3 0 . 8}$ & $\mathbf{8 4 . 6}$ \\
\hline Family History of Substance A & $\mathbf{8 7 . 7}$ & $\mathbf{6 9 . 2}$ \\
\hline Family History of Suicide Attempts & 49.2 \\
\hline
\end{tabular}

Table 2: Patient Information.

\begin{tabular}{|c|c|c|c|c|c|}
\hline Parental Stressors & 1 (not stressful) (\%) & $2 \%$ & 3 (moderate Stress) \% & $4 \%$ & 5 (most stressful)\% \\
\hline Attitude of others regarding having a child with psychiatric problems & 18.2 & 24.2 & 16.7 & 24.2 & 16.7 \\
\hline Financial aspects of having a child with psychiatric problems & 22.7 & 10.6 & 22.7 & 16.7 & 27.3 \\
\hline Confusion or uncertainty about the diagnosis and treatment & 16.7 & 13.6 & 24.2 & 24.2 & 21.2 \\
\hline Safety of the child & 9.1 & 7.6 & 6.1 & 27.3 & 50.0 \\
\hline Safety of the family & 34.8 & 12.1 & 16.7 & 15.2 & 21.2 \\
\hline Balancing the needs of other family members in addition to the child's need & 9.1 & 10.6 & 12.1 & 27.3 & 40.9 \\
\hline Concerns about the child's future & 9.1 & 3.0 & 9.1 & 13.6 & 65.2 \\
\hline Your personal emotional health & 15.2 & 16.7 & 25.8 & 19.7 & 22.7 \\
\hline Inadequate support from family, friends, or other supports & 48.5 & 15.2 & 18.2 & 9.1 & 9.1 \\
\hline The interaction with mental health professionals & 40.9 & 15.2 & 24.2 & 9.1 & 10.6 \\
\hline
\end{tabular}

Table 3: Parental Stressors. 
The average T-score on the PSI-4-SF for these parents was found to be 59.5, which is in the 80th percentile for parent-child dysfunctional relationship. The average score for the domain examining qualities of the child that parents find difficult to manage ranked in the 90th percentile and the score representing the level of distress the parents are experiencing is in the 65 th percentile. The domain which focuses on parent's perception of parent-child dysfunctional interaction was in the 86th percentile, which indicates high stress and recommendation for professional referral [4].

According to our survey for the entire sample of parents: $79 \%$ of the parents are most stressed about the child's future, $77.3 \%$ about safety of the child, $68.2 \%$ balancing the needs of other family members in addition to the child's need for treatment, $45.4 \%$ confusion or uncertainty about the diagnosis and treatment, $44 \%$ financial aspects, $42.4 \%$ their own personal emotional health, $40.9 \%$ stigma, and $36.4 \%$ safety of the family. (Table 3 )

Results for our hypothesis questions are as follows: Figure 1: Whether the number of suicide attempts affects parents concern about the child's future ( $\mathrm{p}=0.42)$; Figure 2: Axis I diagnosis effect on parents' concern about the child's future $(\mathrm{p}=0.2)$; Figure 3 : Family history of mental illness with concern about the child's future $(p=0.9)$; Figure 4 : Stress experienced when interacting with mental health professionals based on Axis I diagnosis $(\mathrm{p}=0.5)$; Figure 5: Whether the parents' education level affects confusion about the child's diagnosis $(\mathrm{p}=0.6)$;
Figure 6: parent educational level with concern about stigma $(\mathrm{p}=1)$. These associations were found to be not statistically significant.

\section{Discussion}

This work represents the first study investigating stress among parents of children admitted to an inpatient psychiatric unit. Our population sample of 66 parents is larger than other pilots which have previously investigated parental stress of outpatient child and adolescent psychiatry patients $[1,6]$. The top stressors among our sample of parents were concerns regarding the future and safety of the child, as well as balancing needs of other family members in addition to the ill family member. This is similar to findings found by Nadkarni and Fristad, as well as Diaz-Caneja, et al. [1,6]. Additionally, this study supports statements that the presence of a mentally ill family member can have a "profoundly disruptive, disorganizing effect" on family life. " The family is faced with the challenge of providing for the needs of the ill member while negotiating the inevitable conflicts among the healthy members, all within an atmosphere fraught with confusion, stigma, and secrecy"[8]. The potential for bias in this study is low, as the surveys were administered by a single technician and investigators did not participate in direct care of the patients or families.

One drawback of our study design was that our standardized instrument, the PSI-IV-SF, was not standardized for children over the age of 12. Therefore, complete information about stress in the parent-child dynamics for children ages 13-17 was not available for

\section{Interaction with Professionals Rating x Axis Diagnosis}

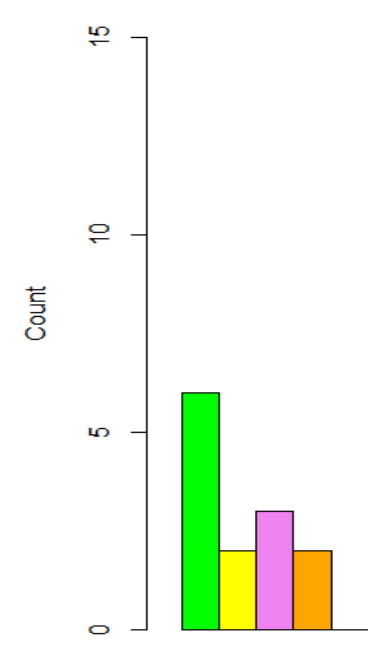

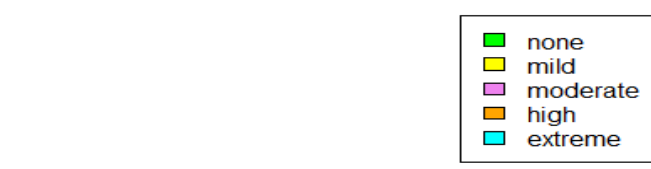

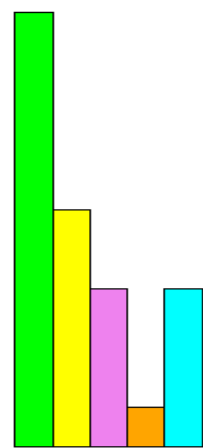

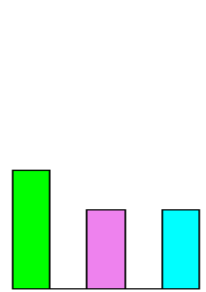

2

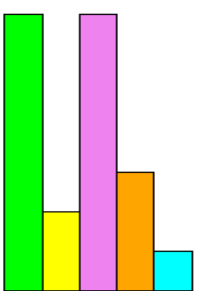

4

Axis I Diagnosis

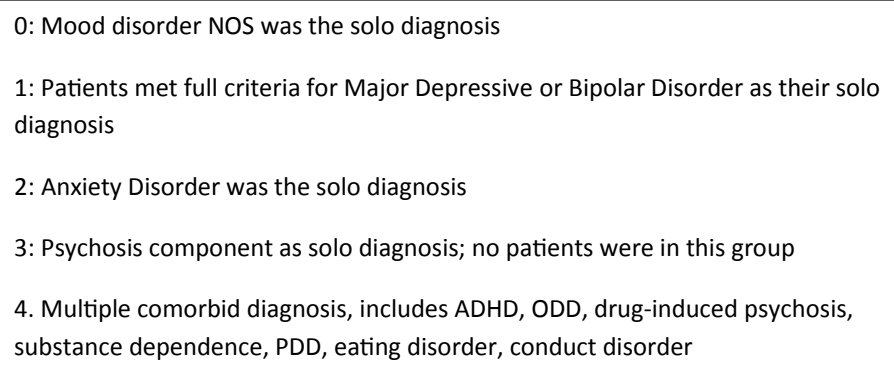

Figure 1: Concern about the future rating $X$ number suicide attempts. 


\section{Stigma Rating $x$ Parents Educational Level}

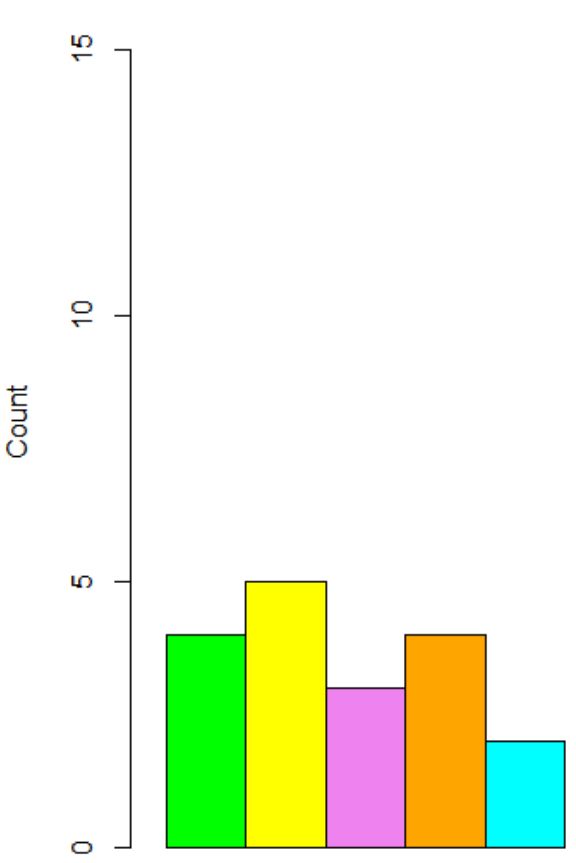

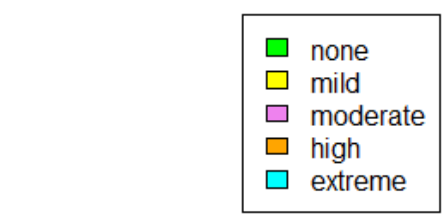

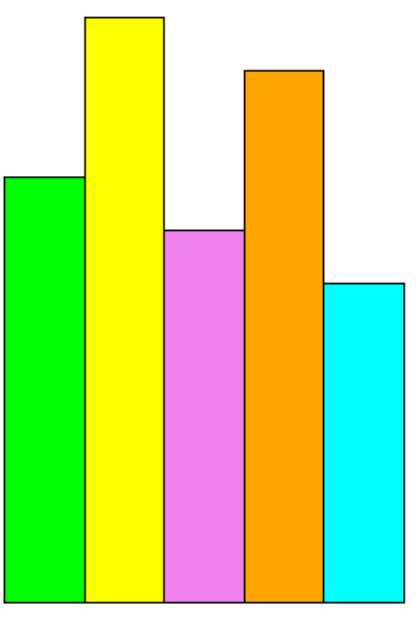

Parents Educational Level

Figure 2: Concern about the future rating $X$ axis 1 Diagnosis.

\section{Concern About The Future Rating x Family history of mental illness}

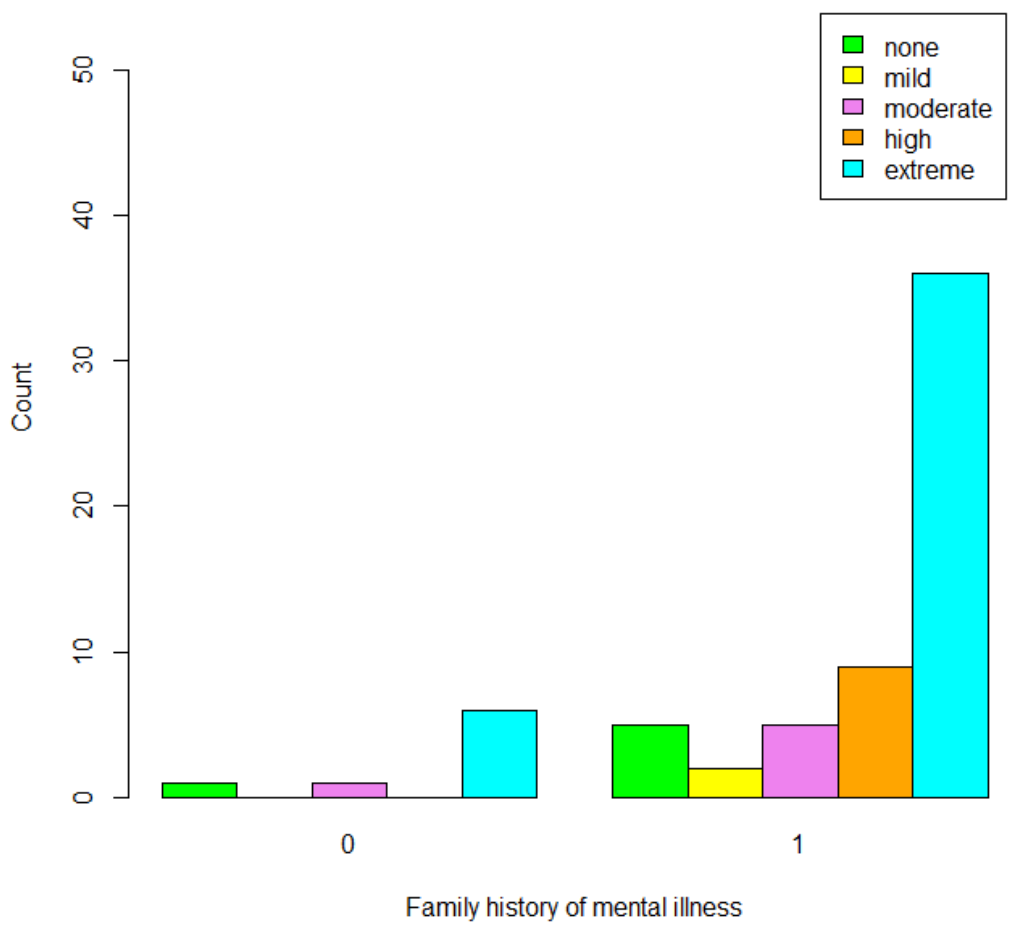

Figure 3: Concern About The Future Rating X Family history of mental illness. 


\section{Confusion About Diagnosis Rating x Parents Educational Level}

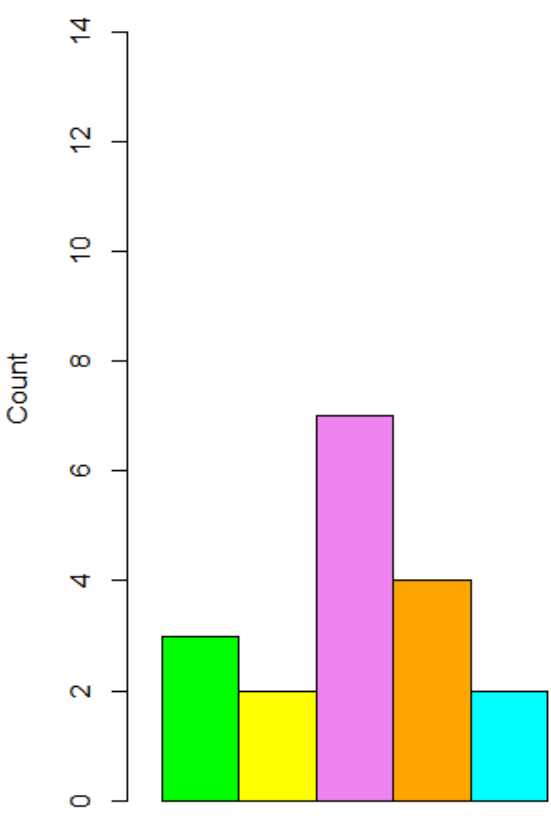

0

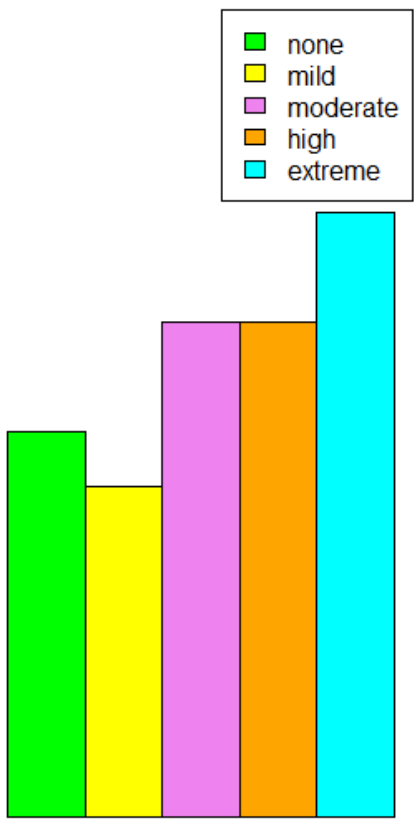

1

Parents Educational Level

Figure 4: Interaction with Professionals Rating X Axis Diagnosis.

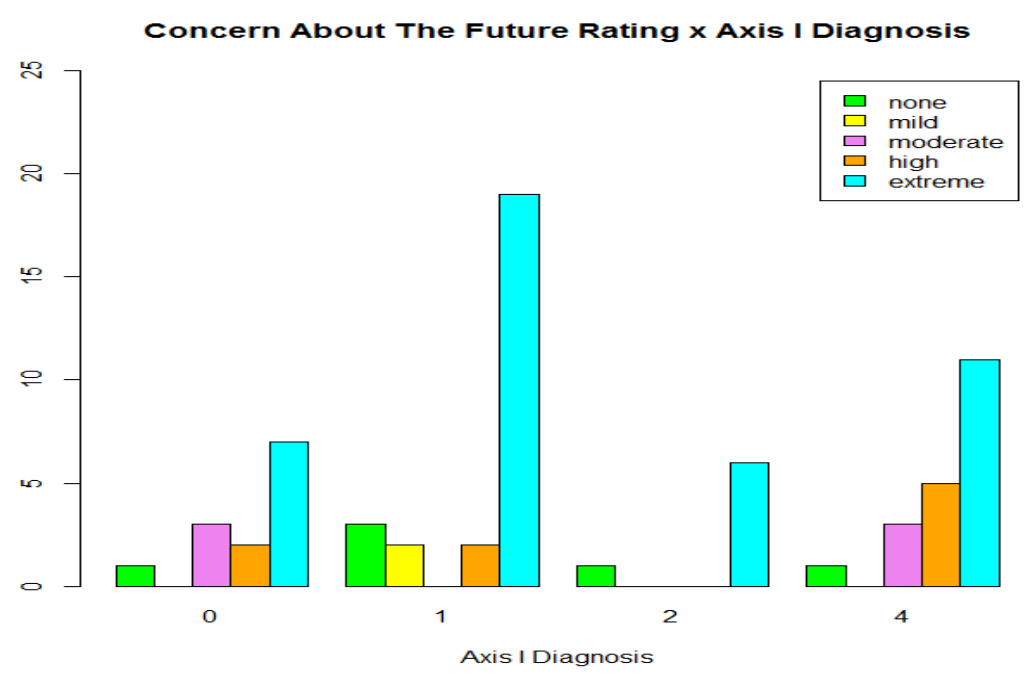

0: Mood disorder NOS was the solo diagnosis

1: Patients met full criteria for Major Depressive or Bipolar Disorder as their solo diagnosis

2: Anxiety Disorder was the solo diagnosis

3: Psychosis component as solo diagnosis; no patients were in this group

4. Multiple comorbid diagnosis, includes ADHD, ODD, drug-induced psychosis, substance dependence, PDD, eating disorder, conduct disorder

Figure 5: Confusuion About Diagnosis Rating X Parents Educational Level. 


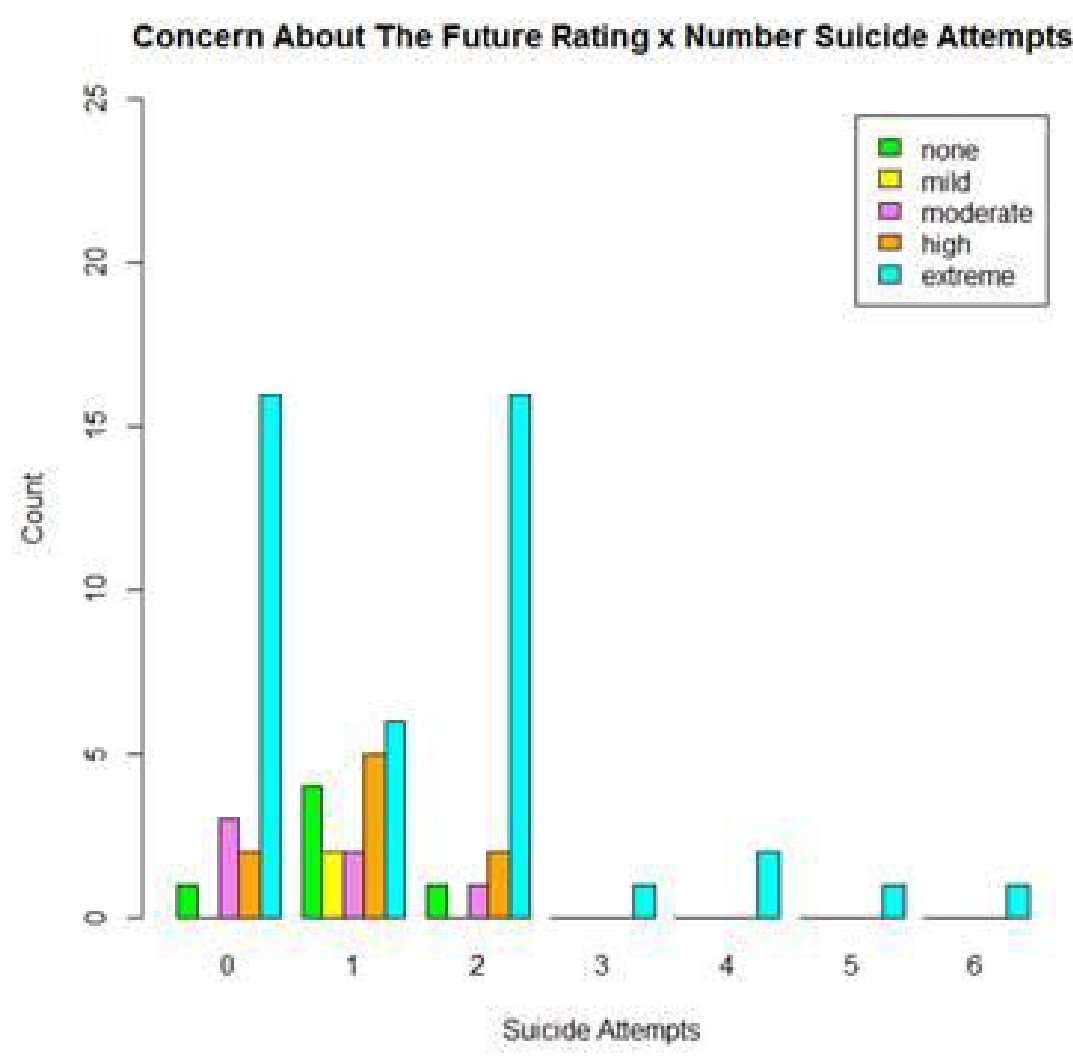

Figure 6: Sigma Rating X Parents Educational Level.

analysis. Researchers for this study did develop a second instrument for exploring the stressors affecting all parents of children in this study, including children over the age of 13 , however this second survey was not a standardized instrument. However, for these parents of children ages 12 and younger, a total stress score could be generated in order to better quantify the overall amount of stress, which was found to be high in the $80^{\text {th }}$ percentile.

For our additional comparative data analysis, it may be that due to a relatively low population size no significant $\mathrm{p}$ values were found. However, our findings were different than we initially expected because they suggest that stress experienced when interacting with mental health professionals does not seem to be associated with Axis I diagnosis, nor does it seem that concern over stigma or confusion regarding diagnosis appears to decrease based on higher parental education level.

As support and psycho-education for parents was previously found to be beneficial to children's prognosis on a medical unit [6], these results could support an intervention where greater emphasis is placed on helping parents develop more positive parent-child relationships, as well as other peer-support and psycho-education programs before the child is discharged from the inpatient psychiatric unit. Based on our study, we would recommend use of a standardized instrument such as the PSI-IV-SF that can indicate which aspects of the parent-child dynamic need focused intervention, whether it is aspects of the parent's expectations, communication with the child, or child temperamental issues.

Possible interventions that can be implemented on an inpatient psychiatric unit are educating unit staff members to develop an empathetic attitude toward caregivers who are often experiencing significant distress due to guilt and lack of confidence regarding caring for their child, balancing their own needs, as well as the family's needs. To promote positive outcome/prognosis in children after inpatient treatment, investing time in the support, shared decision making, psycho-education of their caregivers and interventions to improve parent-child relationships are crucial steps toward recovery. Access to peer support, family therapy, referrals for caregiver mental health care, as well as some basic instruction on fostering healthy communication and limit setting with children are important elements. By helping parents to be engaged in their children's healing process, there is hope that the families can heal as well as their children.

\section{Conclusion}

This pilot study examining stress in parents of children admitted to an inpatient psychiatric unit has a sample size of 66 parents and caregivers. It has been shown previously that parental distress interplays with parent-child dysfunctional interactions, which in turn affects parenting behaviors and potentially worsens child outcomes [7]. Stressors identified in these parents are similar to those identified in other studies of parents of children in outpatient mental health treatment, as well as children admitted to a medical unit. These include financial strain, diminished relationships with social networks, neglect of personal emotional health, concern about the child's future, and confusion related to diagnosis $[1,6]$. Parents with children under the age of 12 who completed the PSI-4-SF, which identifies parent-child systems that are under stress and at risk for developing dysfunctional parenting [7], scored a mean of 59.9. This places the average total stress of these parents in the 80th percentile for this instrument and likely warrants professional intervention, based on other literature used to develop the PSI-4-SF [7]. The parent's perception of parent-child 
Citation: Hissett E, Bilge-Johnson S, McNinch N (2015) An Initial, Prospective Exploration of Specific Stressors for Parents of Children Admitted to an Inpatient Psychiatric Unit. J Psychol Abnorm Child 4: 138. doi:10.4172/2329-9525.1000138

Page 7 of 7

dysfunctional interaction was quite high, in the 86th percentile. This indicates that parents felt significant distress and lack of confidence in their roles as parents, which has also been demonstrated in work with outpatients with mental illness [2].

The results of the survey administered to all parents in the study was that participants' greater stress ratings were reported regarding the safety of the child with mental illness, balancing the needs of other family members in addition to the child needing treatment, and overall concern about the child's future. Parental distress did not seem to be affected by Axis I diagnosis, nor did parental education level affect concern about stigma or confusion related to their child's diagnosis.

\section{References}

1. Nadkarni, Radha B, Fristad, Mary A (2012) Stress and Support for Parents of Youth with Bipolar Disorder. Israel Journal of Psychiatry and Related Sciences 49:104-111.
2. Syed, EhsanU, Zuberi, Saman I (2006) Mental Distress in Mothers of Child Psychiatric Patients. Journal of the College of Physicians and Surgeons 16: 416-419.

3. Mackinaw-Koons B, Fristad, Mary A (2004) Children with Bipolar Disorder: How to Break Down Barriers and Work Effectively Together. Psychol Res Pr35: 481 484

4. Lefley H (1989) Family Burden and Family Stigma in Major Mental Illness. Am Psychol 44: 556-560.

5. Hellander M, Sisson D, Fristad, Mary A (2003) Internet Support of Parents of Children with Early Onset Bipolar Disorder. Bipolar Disorder in Childhood and Early Adolescence 314-329.

6. Diaz-Caneja A, Gledhill J, Weaver T, Nadel S,Garralda E (2005) A Child's Admission to Hospital: A Qualitative Study Examining the Experiences of Parents. The European Society of Intensive Care Medicine 31:1248-1254.

7. Abdin, Richard R, EdD (2012) Parenting Stress Index, Fourth Edition,PAR. Lutz, Florida.

8. Bernheim, Kayla, Anthony Lehman (1985) Working with Families of the Mentally III. The Family's Plight.Norton and Company. 